\title{
Resting state fMRI brain mapping in pediatric supratentorial brain tumors
}

\author{
Ahmed Anwar ${ }^{1,2^{*}}$, Ahmed Radwan ${ }^{3,4}$, Iman Zaky ${ }^{1,2}$, Moatasem El Ayadi ${ }^{5,6}$ and Ayda Youssef $f^{1,2}$
}

\begin{abstract}
Background: Functional mapping of eloquent brain areas is crucial for preoperative planning in patients with brain tumors. Resting state functional MRI (rs-fMRI) allows the localization of functional brain areas without the need for task performance, making it well-suited for the pediatric population. In this study the independent component analysis (ICA) rs-fMRI functional mapping results are reported in a group of 22 pediatric patients with supratentorial brain tumors. Additionally, the functional connectivity (FC) maps of the sensori-motor network (SMN) obtained using ICA and seed-based analysis (SBA) are compared.

Results: Different resting state networks (RSNs) were extracted using ICA with varying levels of sensitivity, notably, the SMN was identified in 100\% of patients, followed by the Default mode network (DMN) (91\%) and Language networks (80\%). Additionally, FC maps of the SMN extracted by SBA were more extensive (mean volume $=25,288.36$ $\mathrm{mm}^{3}$, standard deviation $=13,364.36 \mathrm{~mm}^{3}$ ) than those found on ICA (mean volume $=13,403.27 \mathrm{~mm}^{3}$, standard deviation $=9755.661 \mathrm{~mm}^{3}$ ). This was confirmed by statistical analysis using a Wilcoxon signed rank $t$ test at $p<0.01$.

Conclusions: Results clearly demonstrate the successful applicability of rs-fMRI for localizing different functional brain networks in the preoperative assessment of brain areas, and thus represent a further step in the integration of computational radiology research in a clinical setting.
\end{abstract}

Keywords: Presurgical functional magnetic resonance imaging, Resting-state functional magnetic resonance imaging, Pediatric brain tumors

\section{Background}

Central nervous system (CNS) tumors are among the most common malignancies in childhood and are a leading cause of mortality in this age group [1]. As surgery is the only definitive course of treatment for many of these patients [2], an accurate presurgical functional mapping is crucial to ensure the best possible functional outcome after the surgery. Since the first functional mapping experiments using blood oxygen level dependent (BOLD) functional magnetic resonance imaging (fMRI) were published in 1990 [3], BOLD task-based fMRI has been increasingly used in the clinical setting for presurgical

\footnotetext{
*Correspondence: ahmednagui87@gmail.com

${ }^{1}$ Department of Diagnostic and Interventional Radiology, National

Cancer Institute, Faculty of Medicine, Cairo University, Cairo, Egypt

Full list of author information is available at the end of the article
}

functional mapping. Task-based fMRI (tb-fMRI) entails the performance of a task during scanning; this is typically alternated with a control condition in a symmetrical block design paradigm. Tb-fMRI requires the patient's understanding and cooperation during the examination to acquire useful results [4-6].

Resting-state fMRI (rs-fMRI) was introduced in 1995 by Biswal and colleagues in their seminal work [7]. Rs-fMRI assesses low-frequency fluctuations in the BOLD signal while the subject is at rest, enabling the detection of functionally correlated cortical areas even if they are anatomically remote. This lead to the discovery of the so-called resting-state networks (RSNs) [8-10]. The two most commonly used techniques for rs-fMRI analysis are seed-based analysis (SBA) and independent component analysis (ICA). SBA is performed by placing seeds corresponding to the target 
RSN. It depends on a priori knowledge of expected location of seeds comprising the network, typically a priori atlases or parcellation maps. On the other hand, ICA is a data driven approach which depends on the analysis of signal alterations along the time-course of each voxel and the extraction of correlated activation areas [11]. The main advantages of rs-fMRI over tbfMRI are less time needed for whole brain mapping, as multiple networks can be extracted from one scan, and less cooperation is required from the patient [11].

In this study, the feasibility of functional mapping using ICA rs-fMRI in pediatric patients with supratentorial brain tumors are reported as well as the feasibility of extracting the RSNs most relevant for presurgical mapping, including sensory-motor network (SMN), language network, default mode network (DMN), dorsal attention network (DAN), medial visual and lateral visual networks. Additionally, SBA functional connectivity (FC) maps of the SMN were extracted and compared to the SMN ICA FC maps in the lesioned hemisphere.

\section{Methods}

The present descriptive study was conducted during a period of 18 months, from February 2018 to July 2019. Institutional review board (IRB) approval, in accordance with the Declaration of Helsinki was acquired. A signed written consent form was acquired from the legal guardians of the patients before scanning.

\section{Participants}

This study was carried out on 22 patients; (15 males), age range $2-18$ years (mean age 8.6 years). The study included patients with supratentorial tumors with no history of previous surgical intervention, radiotherapy or chemotherapy or other CNS diseases. Patients were excluded from the study if they had an absolute contraindication to MRI scanning, a degenerative or metabolic disease that may involve the CNS or a multicentric or multifocal brain tumor. Table 1 summarizes the clinical and demographic data of the patients. The most common pathology was Ganglioglioma $(n=4)$ followed by pleomorphic Xanthoastrocytoma $(n=3)$, meningioma $(n=3)$ and dysembryoblastic neuroepithelial tumor (DNET, $n=3$ ). The most common location of the tumor was in

Table 1 Demographics and clinical data of the patients

\begin{tabular}{|c|c|c|c|c|c|c|c|c|}
\hline & Age & Sex & Tumor location & Tumor side & Pathology & WHO grade & Extent of surgery & GA during $M R I$ \\
\hline S01 & 4 & M & Parietal & Left & DNET & । & Debulking & No \\
\hline S02 & 3 & M & Temporal & Left & Anaplastic Ependymoma & IV & Debulking & Yes \\
\hline S03 & 17 & M & Parietal & Right & Anaplastic PXA & III & Excision & No \\
\hline SO4 & 8 & M & Intra-ventricular & Left & Meningioma & 1 & Excision & No \\
\hline S05 & 3 & M & Temporal & Right & Aneurysmal fibrous histiocytoma & & Excision & Yes \\
\hline S06 & 7 & $\mathrm{~F}$ & Frontal & Right & Ganglioglioma & I & Excision & Yes \\
\hline S07 & 10 & $\mathrm{~F}$ & Intra-ventricular & Right & Meningioma & । & Excision & No \\
\hline S08 & 8 & $\mathrm{~F}$ & Temporal & Right & DNET & I & Excision & No \\
\hline S09 & 2 & $\mathrm{~F}$ & Temporal & Right & DNET & । & Excision & Yes \\
\hline S10 & 18 & M & Temporal & Right & Ganglioglioma & । & Excision & No \\
\hline S11 & 16 & $\mathrm{~F}$ & Occipital & Left & Anaplastic PXA & III & Excision & No \\
\hline S12 & 6 & M & Frontal & Right & Pilocytic astrocytoma & I & Debulking & No \\
\hline S13 & 15 & M & Frontal & Right & GBM & IV & Debulking & No \\
\hline S14 & 9 & M & Frontal & Right & Meningioma & I & Excision & No \\
\hline S15 & 16 & M & Frontal & Left & Anaplastic astrocytoma & III & Debulking & No \\
\hline S16 & 8 & M & Temporal & Right & Anaplastic PXA & III & Excision & No \\
\hline S17 & 12 & M & Frontal & Left & Low grade glioma & $\|$ & Debulking & No \\
\hline S18 & 3 & M & Frontal & Right & ETMR & IV & Excision & Yes \\
\hline S19 & 5 & $\mathrm{~F}$ & Temporal & Right & Ganglioglioma & I & Excision & No \\
\hline S20 & 5 & M & Frontal & Right & Ganglioglioma & । & Excision & Yes \\
\hline S21 & 8 & $\mathrm{~F}$ & Fronto-parietal & Right & Anaplastic ependymoma & III & Excision & Yes \\
\hline S22 & 6 & M & Occipital & & Ewing $^{\text {a }}$ & & Excision & Yes \\
\hline
\end{tabular}

GA, general anesthesia; PXA, pleomorphic xanthastrocytoma; DNET, dysembryoblastic neuroepithelial tumor; GBM, glioblastoma multiforme; ETMR, embryonal tumor with multilayered rosettes

${ }^{\text {a }}$ Patient presented with primary occipital bone Ewing Sarcoma with no metastatic lesions 
the frontal lobe $(n=9)$ followed by the temporal lobe $(n=7)$.

\section{Clinical assessment}

Patients were assessed using 5 components of the pediatric NIH stroke scale (NIHSS) [12] the visual, motor, and sensory domains were used without any modification, while the language and dysarthria components were used after translation to Arabic. This modified pediatric NIH stroke scale graded performance on each domain between 0 (normal) and 4 (worst performance), with higher scores indicating worse disability (Additional file 1: Table S1). Patients were assessed preoperatively and at 3 months post-operatively. When functional deficits covered by the Pediatric NIH stroke scale were absent, signs of increased intracranial tension (ICT) or seizures, were recorded.

\section{MR imaging protocol}

MR imaging used a 3 Tesla ( $T$ ) Ingenia MRI scanner (Philips, Eindhoven, the Netherlands) equipped with a 16-channel receive phased-array head coil.

\section{Structural imaging}

3D transverse T1-weighted images covering the whole brain were acquired using a magnetization prepared rapid acquisition gradient echo (MPRAGE), TR/TE/ $\mathrm{FA}=7.9 / 3.5 \mathrm{~ms} / 7^{\circ} \mathrm{FOV}=240 \times 240 \times 250 \mathrm{~mm}$, matrix size $256 \times 256$, voxel size $=0.9 \times 0.9 \times 1 \mathrm{~mm}^{3}$.

\section{Functional imaging}

All fMRI sequences were acquired using an echo-planar imaging (EPI) based T2*-weighted BOLD pulse sequence. Axial slices were acquired in ascending order, $\mathrm{TR} / \mathrm{TE} / \mathrm{FA}=2200 / 30 \mathrm{~ms} / 35^{\circ}, \mathrm{FOV}=112 \times 112 \times 37$ and voxel size of $1.715 \times 1.715 \times 3.3 \mathrm{~mm}^{3}$. We acquired 160 dynamics preceded by 10 initial dummy scans to allow for signal normalization; these dummy scans were discarded on consequent analysis.

\section{Data processing and analysis}

All structural and fMRI images were preprocessed using the CONN toolbox [13]. The default CONN preprocessing pipeline was used, this included the following steps: functional realignment and unwarping; slice timing correction; structural and functional segmentation and normalization to Montreal National Institute (MNI) template, outlier rejection, and functional smoothing. Spatial smoothing was performed using a Gaussian kernel with full width at half maximum of $6.0 \mathrm{~mm}$. Denoising was done using linear regression to remove signal changes related to white matter, cerebrospinal fluid, motion, breathing and cardiac pulsations. Finally, temporal band-pass filtering at $(0-0.9 \mathrm{~Hz})$ and linear detrending were performed.

Preprocessed and denoised data generated by CONN was analyzed using ICA and SBA. The fMRI Software Library's (FSL) [14] Multivariate Exploratory Linear Optimized Decomposition (MELODIC) [15] was used to generate spatio-temporally independent components (ICs) using single-subject ICA. 20 components were generated from each dataset, FSL generated z-maps were converted to uncorrected p-maps using fslmaths. Seed-to-voxel analysis using CONN's first-level analysis was used to generate seed-based functional connectivity uncorrected p-maps.

\section{ICA}

Independent components were examined by two neuroradiologists with 6 and 4 years of experience, while being blinded to the SBA results. The radiologists independently aimed to identify all target networks possible from the data. All networks were identified by the radiologists through their experience and in comparison to published literature on the morphology and the anatomical location of the RSNs $[16,17]$. When disagreement emerged, a final decision was reached by discussion. The language network was defined by the presence of at least 2 of 4 functionally connected components, namely Wernicke's area and/or its right sided homologue (posterior superior temporal gyrus) and Broca's area and/or its right sided homologue (inferior frontal gyrus- pars opercularis and pars triangularis), connectivity in at least 2 of the 4 areas was required to identify the candidate RSN as a language network. When disagreement occurred, the raters reviewed the data together and consensus was achieved.

\section{$S B A$}

Seeds were defined using the contralesional hemisphere labels of the SMN resting-state network atlas included with CONN derived from Human connectome project (HCP) rs-fMRI data $[18,19]$.

\section{Comparing SMN ICA and SBA functional connectivity in the lesioned hemisphere}

ICA and seed-based FC maps for SMN were generated with a $p$ value of less than 0.01 -thus we avoided using direct thresholding- and the resulting images were binarized. Next we applied a mask of the SMN region of interest (ROI) of the diseased hemisphere from the CONN networks atlas after 3-pass dilatation to account for intersubject variability as well as for possible displacement caused by the tumor. 


\section{Statistical analysis}

All statistical calculations were done using Statistical Package for the Social Science (SPSS Inc., Chicago, IL, USA) version 15 for Microsoft Windows. Numerical data were expressed in terms of mean \pm standard deviation, range, frequencies and percentages. Wilcoxon signed rank test was used to compare the number of voxels and volume of the functional connectivity maps of the SMN extracted using ICA and SBA in the diseased hemisphere. Statistical significance of the difference was estimated at $p$ value $\leq 0.01$.

\section{Results}

\section{Clinical outcome}

Pre and 3 months post-operative clinical evaluation of the included patients was performed (Table 2). Nine patients presented with signs of increased ICT or generalized seizures, of whom 8 resolved at 3 months postoperatively, 2 other patients presented with attacks of localized motor seizures without deficits and developed motor deficits post-operatively. Ten patients presented with neurological deficits according to modified NIHSS and one patient died postoperatively. Re-assessment of 9 remaining patients at 3 months postoperatively showed complete resolution or improvement in 6 patients, stationary course in 2 patients and one patient showed worsening scale. One patient presented with a mass at the back of his head felt by the mother that turned out to be Ewing Sarcoma of the occipital bone, he did not develop deficits post-operatively.

\section{ICA}

The SMN was identified in all subjects (100\%) (Fig. 1). At least 2 components of the language network were identified in 17 out of 22 patients (80\%), the 4 components of the language network were identified in 9 out of 22 patients (41\%) (Fig. 2). Additionally, DMN was identified in 20 out of 22 patients (91\%) (Fig. 3), DAN was identified in 17 patients (77\%) (Fig. 4), medial visual network was identified in 12 patients (54\%) (Fig. 5) and lateral visual network was identified in 7 patients (32\%) (Fig. 6).

\section{ICA versus SBA}

SBA maps showed statistically significant difference compared to ICA maps (Wilcoxon signed rank test $p<0.01$ ), with SBA showing larger maps of activation (Table 3) (Fig. 7).

\section{Discussion}

Throughout the past decade, rs-fMRI has been validated in multiple studies as a technique for presurgical brain mapping, where results were highly concordant
Table 2 Clinical evaluation of the patients pre and postoperatively

\begin{tabular}{|c|c|c|}
\hline & Preoperative & Postoperative \\
\hline S01 & Seizures & No deficit \\
\hline SO2 & $\begin{array}{l}\text { Right arm: } 1 \\
\text { Right Leg: } 1 \\
\text { Dysarthria: } 1\end{array}$ & $\begin{array}{l}\text { Right arm: } 2 \\
\text { Right Leg: } 2 \\
\text { Dysarthria: } 2\end{array}$ \\
\hline S03 & Left arm: 1 & Left arm: 0 \\
\hline S04 & Right arm: 1 & Right arm: 0 \\
\hline S05 & $\uparrow I C T$ & No deficit \\
\hline S06 & $\begin{array}{l}\text { Left arm: } 1 \\
\text { Left leg: } 1\end{array}$ & $\begin{array}{l}\text { Left arm: } 1 \\
\text { Left leg: } 1\end{array}$ \\
\hline S07 & $\uparrow I C T$ & No deficit \\
\hline S08 & Seizures & No deficit \\
\hline S09 & Seizures & No deficit \\
\hline S10 & Seizures (temporal lobe epilepsy) & No deficit \\
\hline S11 & Partial hemianopia & Partial hemianopia \\
\hline S12 & Left arm: 2 & No deficit \\
\hline S13 & $\begin{array}{l}\text { Left arm: } 3 \\
\text { Left leg: } 4\end{array}$ & Patient deceased \\
\hline S14 & $\begin{array}{l}\text { Left arm: } 1 \\
\text { Left leg: } 2\end{array}$ & $\begin{array}{l}\text { Left arm: } 1 \\
\text { Left leg: } 1\end{array}$ \\
\hline S15 & Attacks of motor seizures ( right hemiparesis) & Right arm: 1 \\
\hline S16 & $\uparrow I C T$ & No deficit \\
\hline S17 & $\uparrow I C T$ & No deficit \\
\hline S18 & $\begin{array}{l}\text { Left arm:1 } \\
\text { Left leg: } 1\end{array}$ & No deficit \\
\hline S19 & Seizures (temporal lobe epilepsy) & No deficit \\
\hline S20 & Attacks of motor seizures ( left facial seizures) & Left arm: 2 \\
\hline S21 & $\begin{array}{l}\text { Left arm: } 1 \\
\text { Left leg: } 1\end{array}$ & $\begin{array}{l}\text { Left arm: } 0 \\
\text { Left leg: } 1\end{array}$ \\
\hline S22 & Mass felt by mother & No deficit \\
\hline
\end{tabular}

with functional maps extracted through tb-fMRI, intraoperative electro-cortical stimulation (ECS) and even direct visual identification of the motor cortex based on anatomical landmarks [20-22].

Multiple studies compared the reliability of rs-fmri analysis to tb-fMRI, ECS or both. Zhang el al. [23] conducted a study on 17 healthy controls and 4 patients, using SBA rs-fMRI. They were able to localize sensorimotor cortex compared to ECS and performed as well or better than tb-fMRI. In a study on 17 supratentorial glioma patients, SBA rs-fMRI was performed using a seed placed on the hand knob area of the healthy hemisphere. Compared to ECS, the overall sensitivity and specificity values of rs-fMRI were 90.91 and $89.41 \%$, respectively. In the 15 patients who were able to perform tb-fMRI, the sensitivity and specificity values for the hand motor area were 78.57 and $84.76 \%$, respectively. For these 15 patients who underwent both rs-fMRI and tb-fMRI, there was no statistical difference in sensitivity or specificity between these two methods [24]. Another study conducted on 


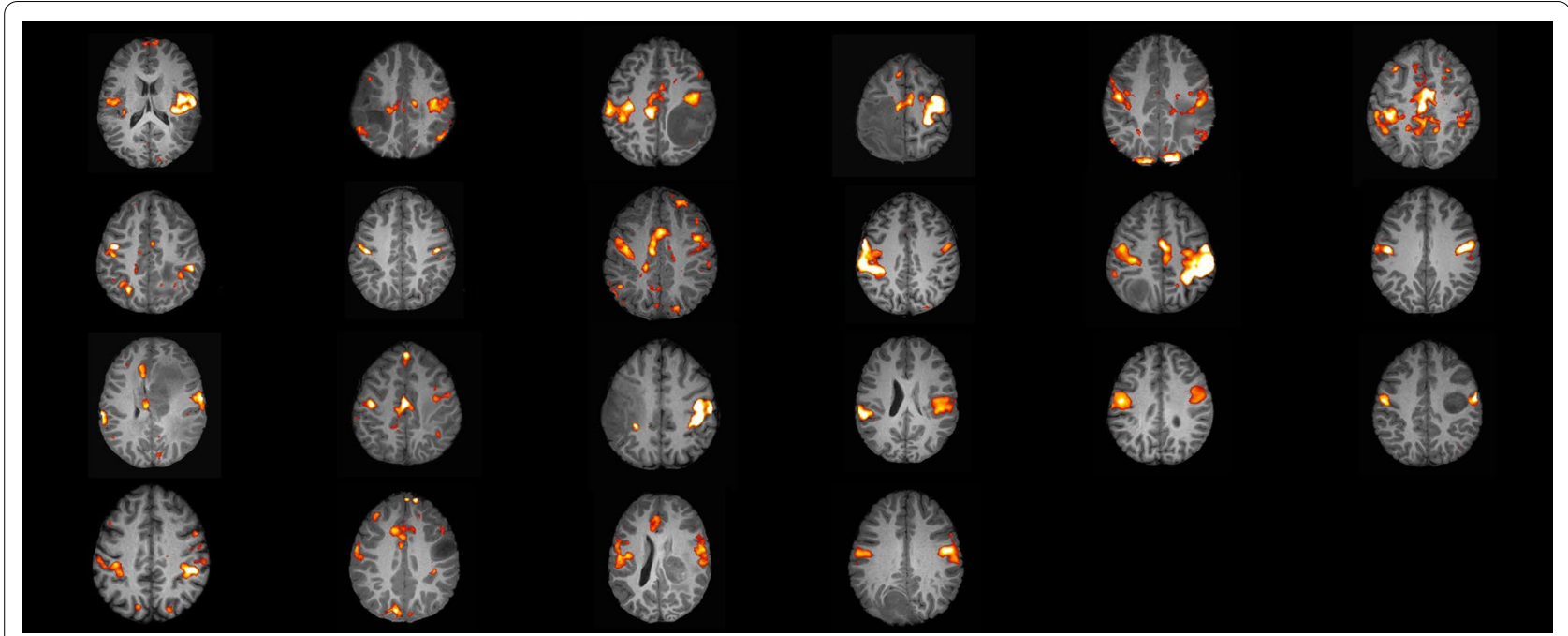

Fig. 1 Activation maps of the components representing SMN in the 22 patients

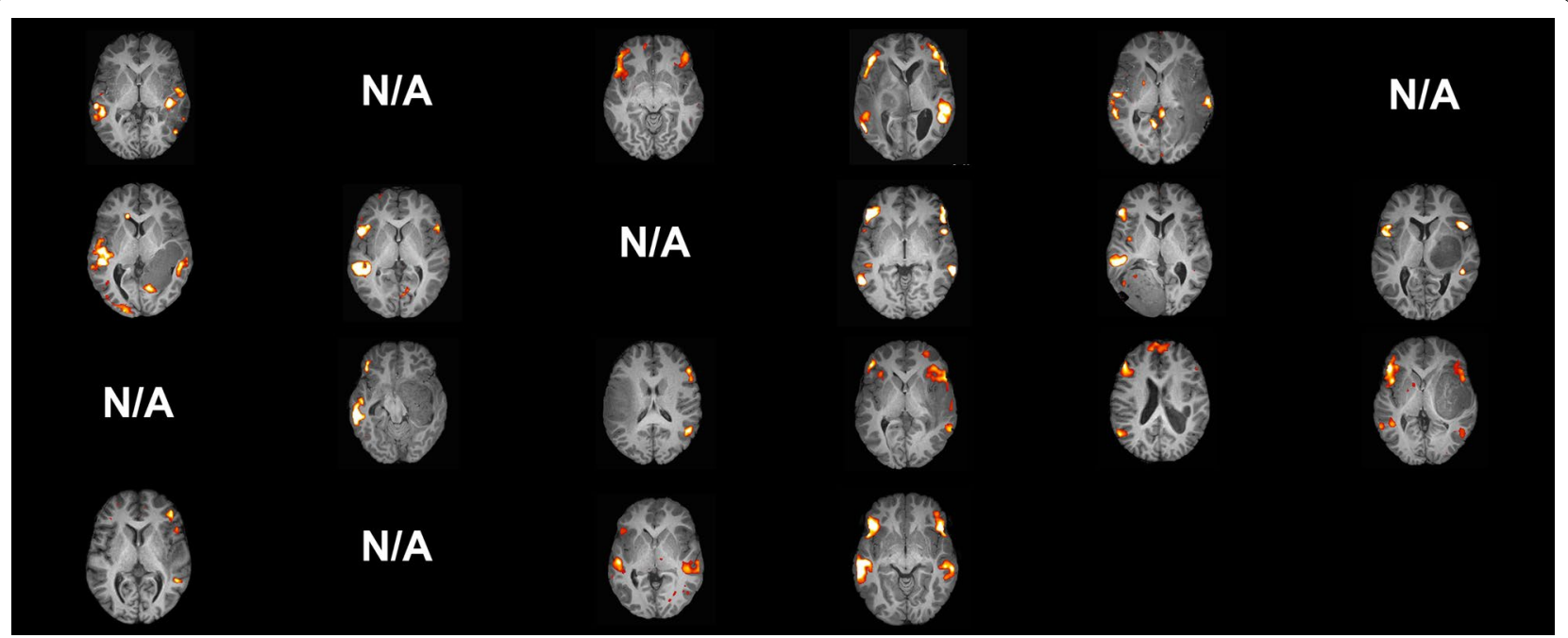

Fig. 2 Activation maps of the components representing language network in 17 patients (N/A, not available)

10 healthy subjects and 25 patients with left sided brain tumors compared tb-fMRI, SBA rs-fMRI and visual anatomical localization of the hand motor found important differences in the locations (i.e., ' $20 \mathrm{~mm}$ ) of the determined hand motor voxels by these three MR imaging methods [20]. In the current study, the sensorimotor cortex was detected using SBA and ICA maps in all patients, the pattern of activation was correlated to the anatomical structure regarding the expected activation area and the two networks showed similar activation areas in the lesional hemisphere.

Several studies used ICA rs-fMRI to extract the SMN, Kokkonen et al. [25] compared tb-fMRI and rs-fMRI using ICA in a group of patients with brain tumors of heterogeneous histopathology as well as a group of healthy controls, they found successfully localized the SMN in both groups, i.e. the brain tumors did not hamper visualization of the SMN in the cases. In the resting state group ICA, both the cases and the controls presented sensorimotor areas similar to the motor task group ICAs and to each other. Schnieider et al. [26] compared ICA rs-fMRI to tb-fMRI in a group of 19 brain tumor patients. They successfully and consistently identified motor cortex by using rs-fMRI and visual assessment by 3 radiologists for all patients. Even when subdividing the motor cortex into 3 segments (hand, face and foot), the sensitivities of 


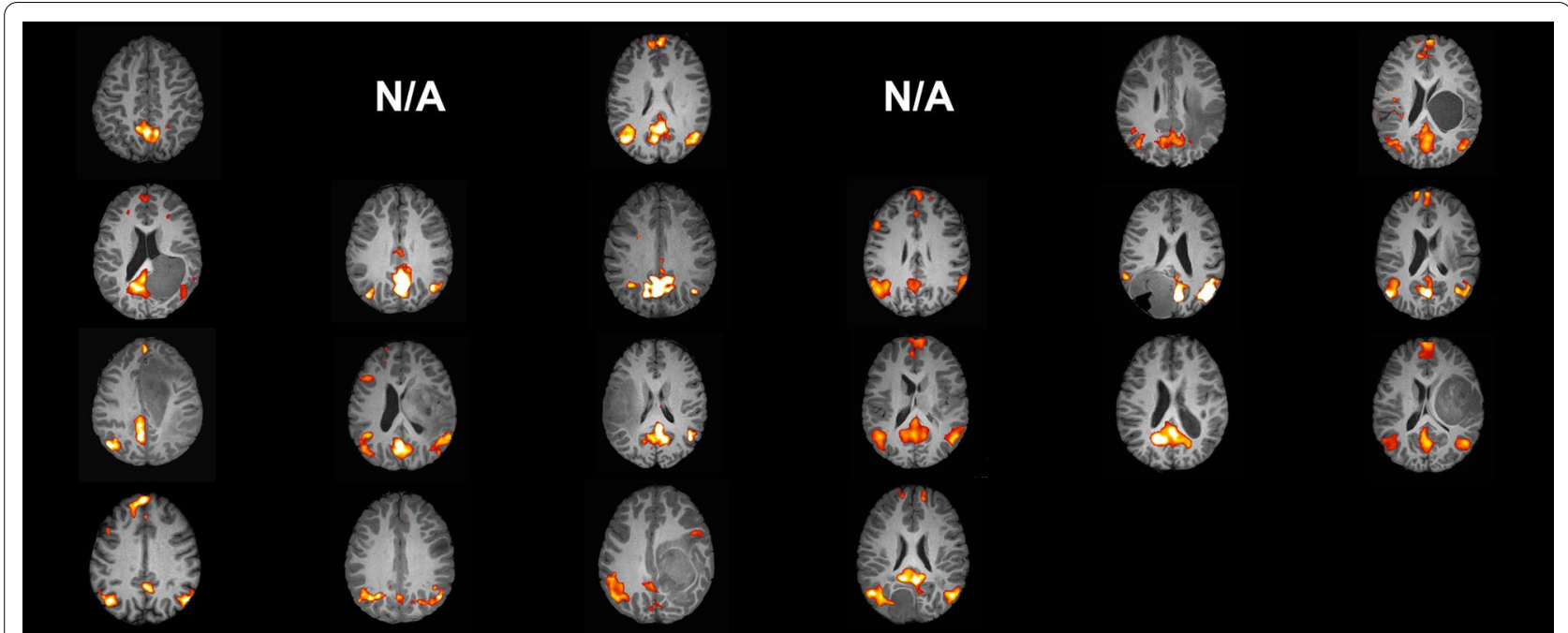

Fig. 3 Activation maps of the components representing DMN in 20 patients (N/A, not available)

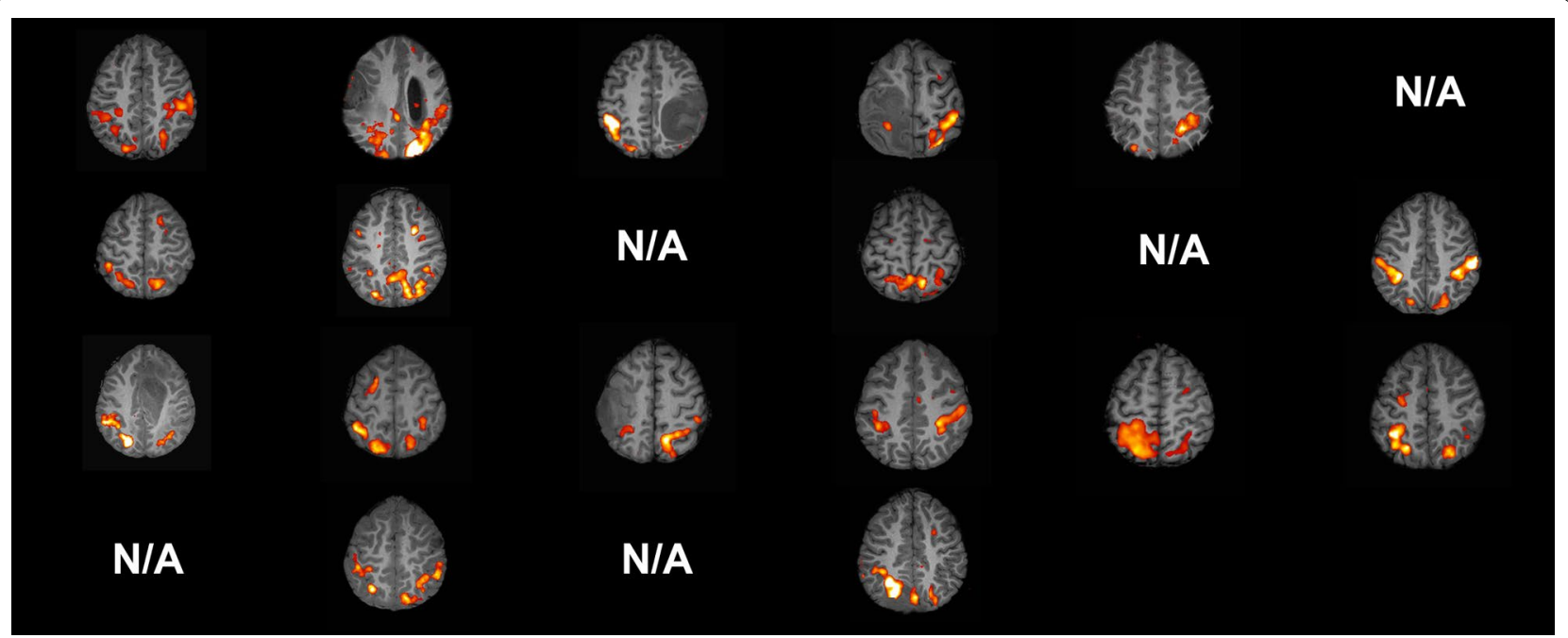

Fig. 4 Activation maps of the components representing DAN in 17 patients (N/A, not available)

rs-fMRI and tb-fMRI were comparable. Another study compared both SBA and ICA RSNs to ECS and found significant correlation between resting-state low frequency fluctuations of BOLD signal and the functional unity of distinct cortical areas confirmed by ECS. Additionally, the ICA method succeeded in detection of the sensori-motor and language networks in 90 (92\%) and 73 (75\%) cases respectively [27]. In the current study, using ICA, the sensorimotor network was identified in $100 \%$ of cases and the 4 components of language network were identified in $41 \%$ of cases. When comparing the SMN FC maps extracted using SBA and ICA, SBA maps were larger than ICA maps at a $p$ value of $\leq 0.01$.
Tie et al. [28] tried detecting ICAs related to language networks in preoperative patients, with 5 experts rating the language networks in ICAs generated from rs-fMRI data of 17 patients. They found moderate agreement between experts, moreover they found that 5 experts agreed upon 1 component most representative of the language network in 10 of the patients, which jumped to 14 upon consensus of only 4 experts. In the present study, using ICA, at least two of the components the language network was detected in 17 out of 22 patients whilst the 4 components of the language network were identified in 9 out of 22 patients. 


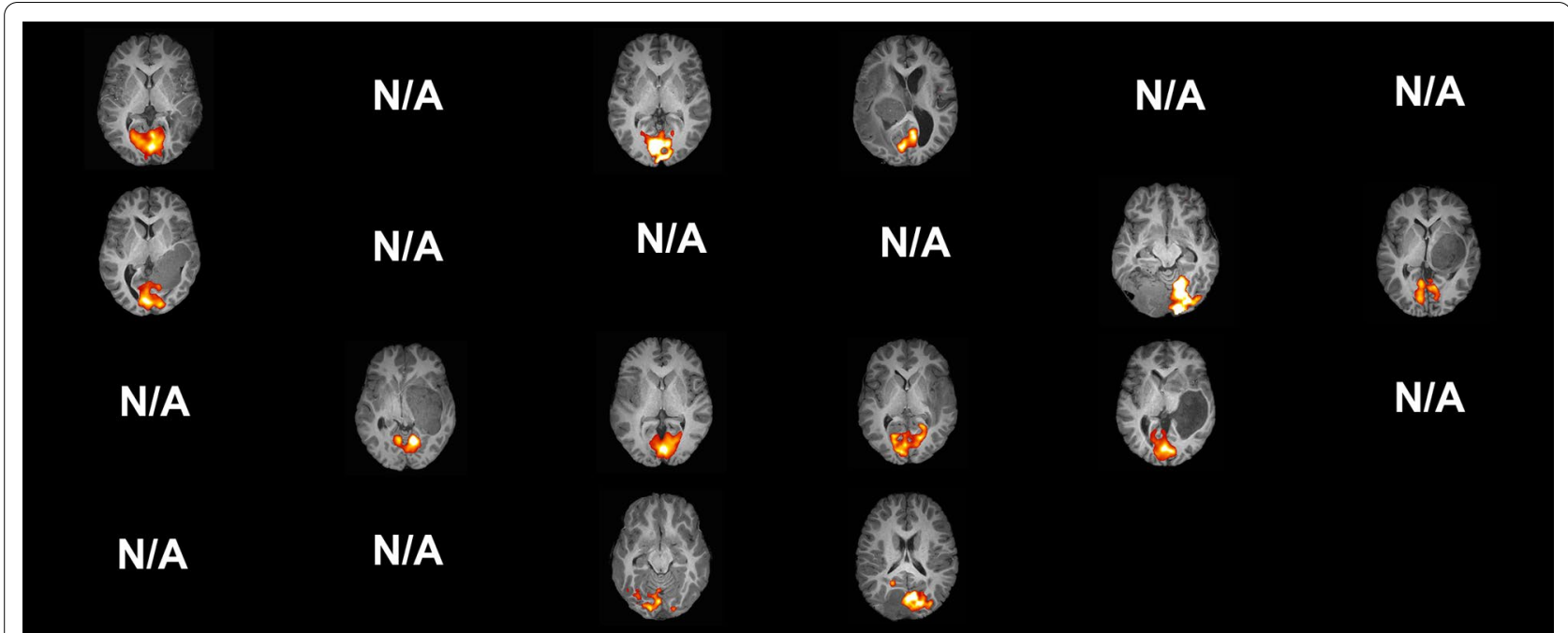

Fig. 5 Activation maps of the components representing medial visual network in 12 patients (N/A, not available)

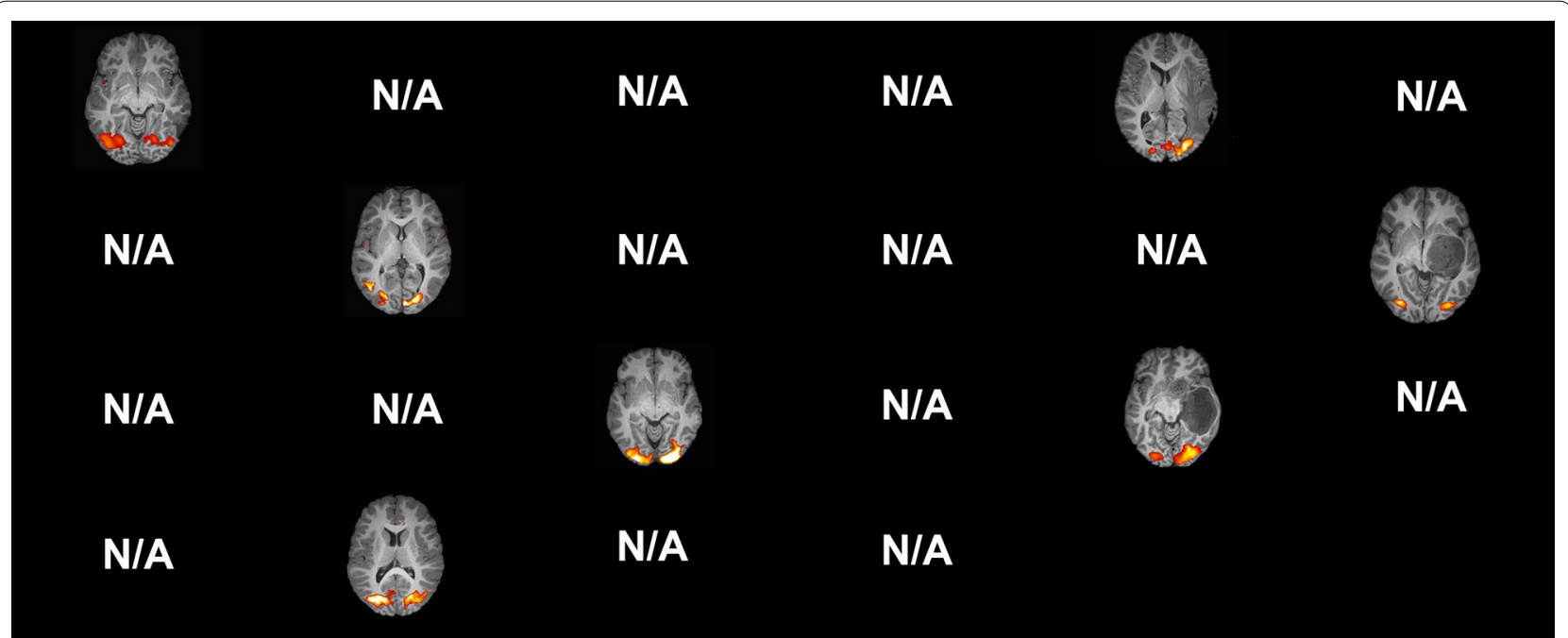

Fig. 6 Activation maps of the components representing lateral visual network in 7 patients (N/A, not available)

The two main methods for ICA RSN recognition are subjective identification of these networks by experts $[23,25]$ and using automated software for detection [29], however the latter may be limited by structural changes caused by tumoral infiltration and mass effect of the tumor on target regions of interest [29]. Additionally, some of the RSNs are more readily reproducible than others, for example, in our study we were able to detect the SMN and DMN more frequently than other RSNs e.g. lateral visual, and DAN.

Thresholding FC maps to segregate actual activity from noise is a debatable subject, especially due to inter-individual differences in activation intensity [30]. A common practice in clinical pre-surgical fMRI is manual thresholding, which is operator dependent [30-32]. Some authors suggested methods to standardize the thresholding of the extracted FC maps including setting a percentage of the maximum intensity detected below which activations are discarded [30]. Even with manual thresholding, radiologists should be aware of the differences in intensity values of FC maps generated by their analysis pipeline. Even with manual thresholding, radiologists should be aware of the difference in the extent of FC maps generated by their analysis pipeline. In our study, the SBA pipeline generated larger FC maps, consequently, more thresholding parameters would be required on these maps for 
Table 3 Volumes of SMN as calculated by ICA and SBA

\begin{tabular}{|c|c|c|c|c|}
\hline Subject number & ICA voxels $\left(\mathrm{mm}^{3}\right)$ & ICA volume $\left(\mathrm{mm}^{3}\right)$ & SBA voxels $\left(\mathrm{mm}^{3}\right)$ & SBA volume $\left(\mathrm{mm}^{3}\right)$ \\
\hline S01 & 4412 & 35,296 & 5873 & 46,984 \\
\hline S02 & 365 & 2920 & 449 & 3592 \\
\hline S03 & 1386 & 11,088 & 3129 & 25,032 \\
\hline S04 & 160 & 1280 & 1188 & 9504 \\
\hline S05 & 1452 & 11,616 & 2364 & 18,912 \\
\hline S06 & 696 & 5568 & 3698 & 29,584 \\
\hline S07 & 607 & 4856 & 29,584 & 29,584 \\
\hline S08 & 3433 & 27,464 & 29,584 & 40,344 \\
\hline S09 & 1219 & 9752 & 1415 & 11,320 \\
\hline S10 & 985 & 7880 & 1879 & 15,032 \\
\hline S11 & 949 & 7592 & 4909 & 39,272 \\
\hline $\mathrm{S} 12$ & 3064 & 24,512 & 5598 & 44,784 \\
\hline $\mathrm{S} 13$ & 1601 & 12,808 & 3413 & 27,304 \\
\hline S14 & 1363 & 10,904 & 1982 & 15,856 \\
\hline S15 & 116 & 928 & 355 & 2840 \\
\hline S16 & 2140 & 17,120 & 4511 & 36,088 \\
\hline S17 & 2520 & 20,160 & 3384 & 27,072 \\
\hline $\mathrm{S} 18$ & 1377 & 11,016 & 1811 & 14,488 \\
\hline S19 & 2534 & 20,272 & 5026 & 40,208 \\
\hline S20 & 116 & 928 & 2402 & 19,216 \\
\hline S21 & 3182 & 25,456 & 2314 & 18,512 \\
\hline S22 & 3182 & 25,456 & 5102 & 40,816 \\
\hline Mean & 1675.409 & $13,403.27$ & 5453.182 & $25,288.36$ \\
\hline Standard deviation & 1219.458 & 9755.661 & 7974.765 & $13,364.36$ \\
\hline
\end{tabular}

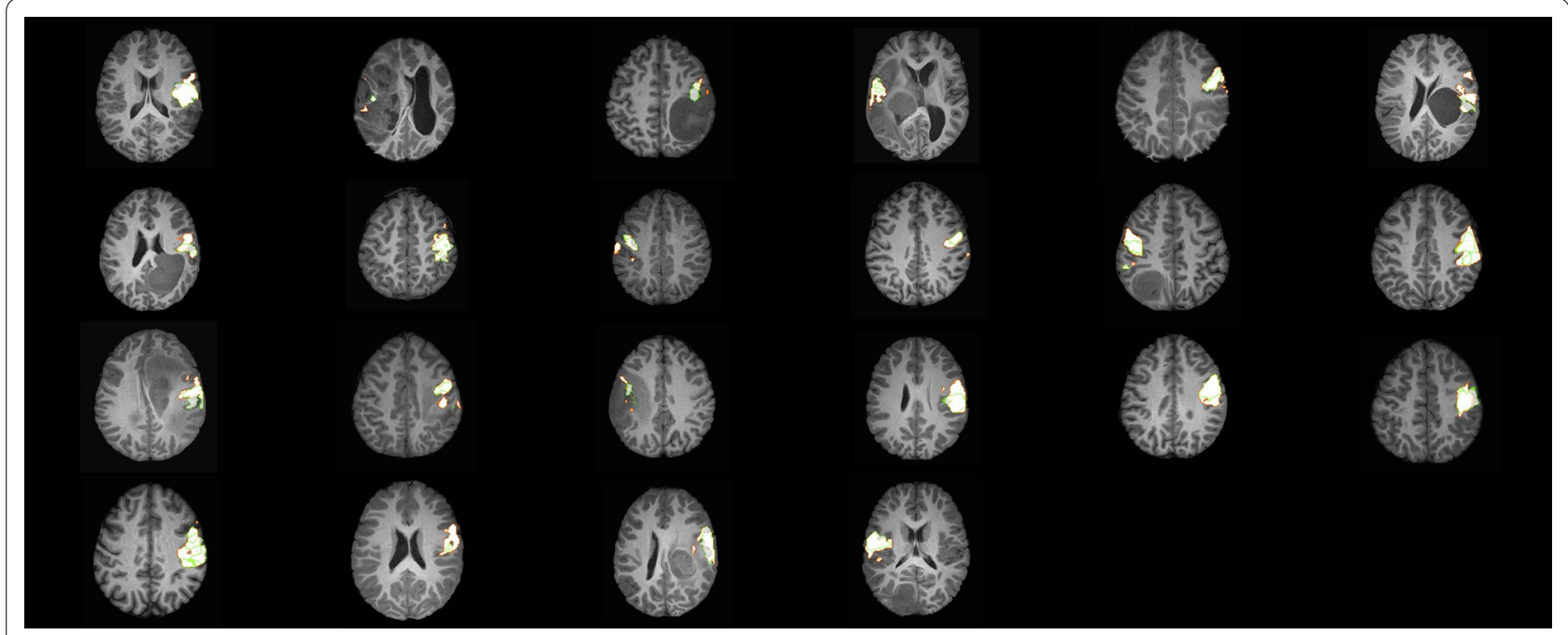

Fig. 7 Motor activationmaps on the lesional hemisphere as conveyed by SBA (red) and ICA (green)

proper delineation of RNSs required for clinical pre-surgical mapping.

A recent meta-analysis of published preoperative tbfMRI studies showed that preoperative fMRI planning -in addition to other advanced imaging techniques- reduced post-operative morbidity [33]. It is safe to project the same conclusions to rs-fMRI, since its maps are closely correlated to those of tb-fMRI, as previously mentioned. 
Nevertheless, the correlation between preoperative rsfMRI and post-operative outcome -especially in pediatric patients-is a difficult subject due to several factors. First, there is no consensus on a clinical functional assessment method for pediatric brain tumors; in fact, many clinical assessment techniques for brain neoplastic lesions rely more on the day-to-day well being rather than gross and fine motor, sensory and language skills e.g. Karnofsky Performance Score, European Organisation for Research and Treatment of Cancer Quality of Life Core Questionnaire..etc. [34]. Other factors include different localization of the neoplastic lesions in relation to the canonical sensori-motor and language eloquent brain areas and their effect on these areas (e,g, displacement Vs infiltration) and surgical approach to the lesions (e.g. debulking Vs excision). The correlation of the tumoral effect on other RSNs e.g. DMN and post-operative cognitive outcome is similarly challenging as in-addition to the previous limitations-the role these networks play in cognitive functions is not yet fully understood [35]. One of the promising methods for better understanding these global and local cerebral RSNs is graph theory analysis, which may also play an important role in the future in the preoperative planning and preservation brain network connectomics through critical node planning [36].

This study was not without limitations, the heterogeneous pathology and location of the tumors -even though reflects actual daily practice- falls short of accounting for their effect on the detection and extent of the extracted RSNs. The definition of the language network did not include some of the ROIs that may play a role in language processing e.g. premotor cortex. Finally, detection of language lateralization using rs-fMRI is still a challenging research subject with promising published data [37, 38].

\section{Conclusions}

In summary, rs-fMRI is a promising tool for pre-surgical planning and predicting the functional prognosis in supratentorial brain tumors. Its importance is specifically higher in the pediatric population. Integration of rs-fMRI in different institutions requires good understanding of the basic concepts and technical aspects of the examination as well as adequate knowledge on how to analyze and interpret the results.

\footnotetext{
Abbreviations

BOLD: Blood oxygen level dependent; CNS: Central nervous system; DAN: Dorsal attention network; DMN: Default mode network; EPI: Echo-planar imaging; FA: Flip angle; FC: Functional connectivity; fMRI: Functional magnetic resonance imaging; FOV: Field of view; FSL: FMRI Software Library's; HCP: Human connectome project; ICA: Independent component analysis; ICT: Intracranial tension; IRB: Institutional Review Board; MELODIC: Multivariate exploratory linear optimized decomposition; MNI: Montreal National Institute; MPRAGE: Magnetization prepared rapid acquisition gradient echo; NIHSS: NIH Stroke Scale; ROI: Region of interest; rs-fMRI: Resting-state fMRI; RSNs: Resting-state
}

networks; SBA: Seed-based analysis; SMN: Sensori-Motor Network; T: Tesla; tbfMRI: Task-based fMRI; TE: Echo time; TR: Repitition time.

\section{Supplementary Information}

The online version contains supplementary material available at https://doi. org/10.1186/s43055-022-00713-3.

Additional file 1: Table S1. Chart used for scoring modified pediatric NIH stroke scale.

\section{Acknowledgements}

Not applicable.

\section{Authors' contributions}

AA: conception and design of the work, acquisition and analysis of data, interpretation of data, drafted the work. AR: conception and design of the work, analysis of data, interpretation of data, revised the work. IZ: conception and design of the work and revised the work. MEA: conception and design of the work and revised the work. AY: conception and design of the work and revised the work. All authors read and approved the final manuscript.

Funding

No funding was received for this research.

\section{Availability of data and materials}

The datasets used and/or analysed during the current study are available from the corresponding author on reasonable request.

\section{Declarations}

Ethics approval and consent to participate

The present study was conducted with institutional review board (IRB) approval, in accordance with the Declaration of Helsinki.

\section{Consent for publication}

A signed written consent form was acquired from the legal guardians of the patients before scanning.

\section{Competing interests}

The authors declare that they have no competing interests.

\section{Author details}

${ }^{1}$ Department of Diagnostic and Interventional Radiology, National Cancer Institute, Faculty of Medicine, Cairo University, Cairo, Egypt. ${ }^{2}$ Department of Radiology, Children's Cancer Hospital (CCHE-57357), Cairo, Egypt. ${ }^{3}$ Department of Imaging and Pathology, Translational MRI, KU Leuven, Leuven, Belgium. ${ }^{4}$ Department of Neurosciences, Leuven Brain Institute (LBI), KU Leuven, Leuven, Belgium. ${ }^{5}$ Department of Pediatric Oncology, National Cancer Institute, Cairo University, Giza, Egypt. ${ }^{6}$ Department of Pediatric Oncology, Children's Cancer Hospital (CCHE-57357), Cairo, Egypt.

Received: 15 December 2021 Accepted: 2 February 2022

Published online: 07 February 2022

\section{References}

1. Ostrom QT, Patil N, Cioffi G et al (2020) CBTRUS statistical report: primary brain and other central nervous system tumors diagnosed in the United States in 2013-2017. Neuro Oncol 22:IV1-IV96. https://doi.org/10.1093/ neuonc/noaa200

2. Souweidane MM (2009) The evolving role of surgery in the management of pediatric brain tumors. J Child Neurol 24:1366-1374. https://doi.org/ $10.1177 / 0883073809342274$ 
3. Ogawa S, Lee TM, Kay AR, Tank DW (1990) Brain magnetic resonance imaging with contrast dependent on blood oxygenation. Proc Natl Acad Sci USA 87:9868-9872. https://doi.org/10.1073/pnas.87.24.9868

4. Tyndall AJ, Reinhardt J, Tronnier V et al (2017) Presurgical motor, somatosensory and language fMRI: technical feasibility and limitations in 491 patients over 13 years. Eur Radiol 27:267-278. https://doi.org/10.1007/ s00330-016-4369-4

5. Tie Y, Suarez RO, Whalen S et al (2009) Comparison of blocked and eventrelated fMRI designs for pre-surgical language mapping. Neuroimage 47:T107-T115. https://doi.org/10.1016/j.neuroimage.2008.11.020

6. Jack CR, Thompson RM, Butts RK et al (1994) Sensory motor cortex: correlation of presurgical mapping with functional MR imaging and invasive cortical mapping. Radiology 190:85-92. https://doi.org/10.1148/radio logy.190.1.8259434

7. Biswal B, Yetkin FZ, Haughton VM, Hyde JS (1995) Functional connectivity in the motor cortex of resting human brain using echo-planar MRI. Magn Reson Med 34:537-541. https://doi.org/10.1002/MRM.1910340409

8. Van Dijk KRA, Hedden T, Venkataraman A et al (2010) Intrinsic functional connectivity as a tool for human connectomics: theory, properties, and optimization. J Neurophysiol 103:297-321. https://doi.org/10.1152/jn. 00783.2009

9. van den Heuvel MP, Hulshoff Pol HE (2010) Exploring the brain network: a review on resting-state fMRI functional connectivity. Eur Neuropsychopharmacol 20:519-534

10. Biswal BB, Mennes M, Zuo XN et al (2010) Toward discovery science of human brain function. Proc Natl Acad Sci USA 107:4734-4739. https:// doi.org/10.1073/pnas.0911855107

11. Roland JL, Hacker CD, Leuthardt EC (2020) A review of passive brain mapping techniques in neurological surgery. Neurosurgery 88:15-24. https:// doi.org/10.1093/neuros/nyaa361

12. Ichord RN, Bastian R, Abraham L et al (2011) Interrater reliability of the Pediatric National Institutes of Health Stroke Scale (PedNIHSS) in a multicenter study. Stroke 42:613-617. https://doi.org/10.1161/STROKEAHA. 110.607192

13. Whitfield-Gabrieli S, Nieto-Castanon A (2012) Conn: a functional connectivity toolbox for correlated and anticorrelated brain networks. Brain Connect 2:125-141. https://doi.org/10.1089/BRAIN.2012.0073

14. Jenkinson M, Beckmann CF, Behrens TEJ et al (2012) FSL. Neuroimage 62:782-790. https://doi.org/10.1016/J.NEUROIMAGE.2011.09.015

15. Beckmann CF, Smith SM (2004) Probabilistic independent component analysis for functional magnetic resonance imaging. IEEE Trans Med Imaging 23:137-152. https://doi.org/10.1109/TMI.2003.822821

16. Cole DM, Smith SM, Beckmann CF (2010) Advances and pitfalls in the analysis and interpretation of resting-state FMRI data. Front Syst Neurosci. https://doi.org/10.3389/FNSYS.2010.00008

17. Xuan Y, Meng C, Yang Y et al (2012) Resting-state brain activity in adult males who stutter. PLoS ONE. https://doi.org/10.1371/JOURNAL.PONE. 0030570

18. NITRC: CONN: functional connectivity toolbox: file release notes and changelog. https://www.nitrc.org/frs/shownotes.php?release_id=4242. Accessed 10 Sep 2021

19. González-Roldán AM, Terrasa JL, Sitges C et al (2020) Age-related changes in pain perception are associated with altered functional connectivity during resting state. Front Aging Neurosci. https://doi.org/10.3389/ FNAGI.2020.00116

20. Hou BL, Bhatia S, Carpenter JS (2016) Quantitative comparisons on hand motor functional areas determined by resting state and task BOLD fMRI and anatomical MRI for pre-surgical planning of patients with brain tumors. Neurolmage Clin 11:378-387. https://doi.org/10.1016/j.nicl.2016. 03.003

21. Haufe S, DeGuzman P, Henin S et al (2018) Elucidating relations between $\mathrm{fMRI}, \mathrm{ECOG}$, and EEG through a common natural stimulus. Neuroimage 179:79-91. https://doi.org/10.1016/j.neuroimage.2018.06.016

22. Hacker CD, Snyder AZ, Pahwa M et al (2017) Frequency-specific electrophysiologic correlates of resting state fMRI networks. Neuroimage 149:446-457. https://doi.org/10.1016/j.neuroimage.2017.01.054

23. Zhang D, Johnston JM, Fox MD et al (2009) Preoperative sensorimotor mapping in brain tumor patients using spontaneous fluctuations in neuronal activity imaged with functional magnetic resonance imaging: Initial experience. Neurosurgery. https://doi.org/10.1227/01.NEU.00003 50868.95634.CA
24. Qiu T et al (2014) Localizing hand motor area using resting-state fMRI: validated with direct cortical stimulation. Acta Neurochir (Wien) 156:2295-2302. https://doi.org/10.1007/s00701-014-2236-0

25. Kokkonen SM, Nikkinen J, Remes J et al (2009) Preoperative localization of the sensorimotor area using independent component analysis of restingstate fMRI. Magn Reson Imaging 27:733-740. https://doi.org/10.1016/j. mri.2008.11.002

26. Schneider FC, Pailler M, Faillenot I et al (2016) Presurgical assessment of the sensorimotor cortex using resting-state fMRI. Am J Neuroradiol 37:101-107. https://doi.org/10.3174/ajnr.A4472

27. Cochereau J, Deverdun J, Herbet $\mathrm{G}$ et al (2016) Comparison between resting state fMRI networks and responsive cortical stimulations in glioma patients. Hum Brain Mapp 37:3721-3732. https://doi.org/10.1002/hbm. 23270

28. Tie Y, Rigolo L, Norton IH et al (2014) Defining language networks from resting-state fMRI for surgical planning — a feasibility study. Hum Brain Mapp 35:1018-1030. https://doi.org/10.1002/hbm.22231

29. Lu J, Zhang H, Hameed NUF et al (2017) An automated method for identifying an independent component analysis-based language-related resting-state network in brain tumor subjects for surgical planning. Sci Rep 71(7):1-16. https://doi.org/10.1038/s41598-017-14248-5

30. Rosazza C, Aquino D, D'Incerti L et al (2014) Preoperative mapping of the sensorimotor cortex: comparative assessment of task-based and restingstate fMRI. PLoS ONE 9:e98860. https://doi.org/10.1371/journal.pone. 0098860

31. Blatow M, Reinhardt J, Riffel K et al (2011) Clinical functional MRI of sensorimotor cortex using passive motor and sensory stimulation at 3 tesla. J Magn Reson Imaging 34:429-437. https://doi.org/10.1002/jmri.22629

32. Håberg A, Kvistad KA, Unsgård G et al (2004) Preoperative blood oxygen level-dependent functional magnetic resonance imaging in patients with primary brain tumors: clinical application and outcome. Neurosurgery 54:902-915. https://doi.org/10.1227/01.NEU.0000114510.05922.F8

33. Luna LP, Sherbaf FG, Sair HI et al (2021) Can preoperative mapping with functional MRI reduce morbidity in brain tumor resection? A systematic review and meta-analysis of 68 observational studies. Radiology. https:// doi.org/10.1148/radiol.2021204723

34. Nayak L, Deangelis LM, Brandes AA et al (2017) The Neurologic Assessment in Neuro-Oncology (NANO) scale: a tool to assess neurologic function for integration into the response assessment in neuro-oncology (RANO) criteria. Neuro Oncol 19:625-635. https://doi.org/10.1093/neuonc/nox029

35. Smith V, Mitchell DJ, Duncan J (2018) Role of the default mode network in cognitive transitions. Cereb Cortex 28:3685-3696. https://doi.org/10. 1093/CERCOR/BHY167

36. Aerts H, Schirner M, Dhollander T et al (2020) Modeling brain dynamics after tumor resection using the virtual brain. Neuroimage. https://doi. org/10.1016/j.neuroimage.2020.116738

37. Liu H, Stufflebeam SM, Sepulcre J et al (2009) Evidence from intrinsic activity that asymmetry of the human brain is controlled by multiple factors. Proc Natl Acad Sci USA 106:20499-20503. https://doi.org/10.1073/ pnas.0908073106

38. Zhu L, Fan Y, Zou Q et al (2014) Temporal reliability and lateralization of the resting-state language network. PLoS ONE 9:e85880. https://doi.org/ 10.1371/journal.pone.0085880

\section{Publisher's Note}

Springer Nature remains neutral with regard to jurisdictional claims in published maps and institutional affiliations. 